\title{
Distribuição Espacial da Pobreza no Paraná
}

\author{
Leandro Nunes Soares da Silva ${ }^{1}$ \\ Murilo José Borges² \\ José Luiz Parré ${ }^{3}$
}

\begin{abstract}
Resumo: Embora haja crescente importância do estudo dos temas relacionados à pobreza, tanto no meio acadêmico, como na política com as promessas incisivas de erradicação da pobreza e o lançamento de programas de transferência de renda, os estudos de pobreza utilizando-se da técnica de análise espacial ainda estão incipientes. Dessa forma, esse artigo tem por objetivo analisar a distribuição espacial da pobreza paranaense em nível municipal, juntamente com os seus possíveis determinantes. Para tanto, utilizou-se o instrumental da Econometria Espacial. A Análise Exploratória de Dados Espaciais revelou a presença de autocorrelação espacial da pobreza entre os municípios estudados, de modo que a pobreza em um município é explicada, em parte, pela pobreza nos municípios vizinhos, além de evidenciar que o Estado paranaense apresenta uma região significativa (Centro-Sul) em que predomina a alta incidência de família pobres e duas regiões menores que apresentam pequena proporção de pobres. Além disso, os resultados econométricos demonstraram a necessidade de incorporar os efeitos da defasagem espacial para um melhor ajuste do modelo a ser estimado. Os resultados, como se esperava, indicaram que quanto maior a renda média e a urbanização, menores são a incidência de pobreza, ao contrário do que ocorre com o Índice de Gini. Por fim, a variável de defasagem espacial da pobreza apresentou sinal positivo, indicando que a pobreza em um determinado município é explicada positivamente pela incidência de pobreza nos municípios vizinhos, ou seja, quanto maior a taxa de pobreza na vizinhança, ceteris paribus, maior a taxa de pobreza no referido município.
\end{abstract}

Palavras-chave: Pobreza; Análise Espacial; Economia Paranaense.

JEL: Co1, C49, I32, I39 e Z13

1. Graduado em Ciências Econômicas na Universidade Estadual de São Paulo - Unesp Araraquara (2012) e mestrando em Ciências Econômicas na Universidade Estadual de Maringá (UEM). E-mail: ssnleandro@ yahoo.com.br

2. Professor na Faculdade Cidade Verde (FCV) - Maringá. Graduado em Economia na Universidade Estadual de Maringá (UEM) e mestrado em Ciências Econômicas na UEM. E-mail: murilo_borges@hotmail.com

3. Doutor em Economia Aplicada (ESALQ /USP) - Professor Titular do Departamento de Economia na Universidade Estadual de Maringá (UEM). E-mail: jlparre@uem.br 


\title{
Spatial Distribution of Poverty in Paraná
}

\begin{abstract}
Although there is a growing importance of the study of issues related to poverty, both in academia as in politics, with policy promises incisively aiming the poverty eradication, with social programs and the beginning of income transfers, poverty studies using the technique of spatial analysis still are incipient. Thus, this article aims to analyze the spatial distribution of poverty in Parana municipal level, along with their possible determinants. For this purpouse, we used the instrumental of Spatial Econometrics. The Exploratory Spatial Data Analysis revealed the presence of spatial poverty among the cities studied, so that poverty in a municipality is explained partly by poverty in neighboring municipalities, in addition to showing that the state of Paraná center-south region presents a high incidence significant of poor families, as other two smaller regions that present a proportion of poverty in a smaller degree. Moreover, the econometric results show the need to incorporate the effects of spatial lag for a better fit of the model to be estimated. The results indicated that as expected the higher average incomes and urbanization, the lower the incidence of poverty, unlike what happens with the Gini Index. Finally, the variable spatial lag of poverty presented positive sign, indicating that poverty in a given municipality is positively explained by the incidence of poverty in neighboring municipalities, ie the higher the poverty rate in the neighborhood, ceteris paribus, the higher the poverty rate in that municipality.
\end{abstract}

Keywords: Poverty; Spatial Analysis; Paranaense Economy.

JEL: Co1, C49, I32, I39 e Z13

\section{Introdução}

Os temas relacionados à pobreza estão na pauta do dia dos governantes brasileiros, tanto é que foram um dos principais temas nas últimas campanhas presidenciais. Fora isso, as políticas públicas de distribuição de renda e erradicação da pobreza, como o Programa Bolsa Família estão entre as políticas públicas mais conhecidas pela população brasileira. Não obstante, esse programa tem sido copiado por outros países e é considerado um exemplo de combate à pobreza.

Ademais, os dados oficiais apontam queda nos índices de pobreza no Brasil partir 2003 em diante. Tal fato é acompanhado, nesse último período por queda na desigualdade de renda e por aumentos reais na renda média da população, sobretudo nos estratos da população mais pobres (IPEA, 2010). Antes disso, em 2000, o Brasil assinou a Declaração do Milênio junto à Organização das Nações Unidos (ONU) no qual se comprometeu a reduzir pela metade a extrema pobreza até 2015. Embora cumprido a meta da referida Declaração em menos de 10 anos, há ainda um árduo caminho para o Brasil erradicar a pobreza que possui uma intensidade maior na área rural e no norte do país. 
O presente artigo tem por objetivo analisar a incidência da pobreza nos 399 municípios de um estado considerado rico e com a sexta maior população do Brasil: o Paraná. Pelo fato de ser um estado com alta renda per capita e baixos índices de pobreza, quando comparados com o restante do país, há poucos estudos de pobreza para o Paraná. Para tanto, será calculado o índice I de Moran para verificar se há dependência espacial entre os municípios e, caso positivo, será estimada a pobreza com a metodologia da econometria espacial e utilizando-se como variáveis explicativas o índice de Gini, a Renda Média, Grau de Urbanização, além das variáveis defasadas espacialmente de cada uma das variáveis explicativas e da variável dependente.

O artigo é composto por cinco partes, começando por essa introdução. $\mathrm{Na}$ segunda parte do artigo serão descritos os conceitos de pobreza e suas diferentes dimensões, são elas a unidimensional, em que a pobreza está relacionada às variáveis econômicas como a renda, sendo esta dimensão escolhida por este trabalho, e a multidimensional em que além das variáveis econômicas consideram-se também variáveis culturais e políticas. A terceira parte discutirá a metodologia do trabalho empregado que se refere à análise dos dados espaciais e aos modelos econométricos espaciais, além da descrição dos dados utilizados. Na quarta parte deste artigo estão os resultados da análise espacial da pobreza e os modelos econométricos que procuram explicar seus determinantes. Por fim, na quinta parte são apresentadas as considerações finais, enfatizando seu objetivo primeiro: analisar a distribuição espacial da pobreza entre os municípios paranaenses, juntamente com os possíveis determinantes da mesma.

\section{Referencial teórico}

A identificação dos pobres e sua quantificação constituem um campo de pesquisa antigo. Em 1901, Joseph Rowntree publicou na Inglaterra um estudo "Poverty, a study of town life" em que utilizou informações como salários, necessidades nutricionais e habitação de modo a distinguir as famílias em situação de pobreza, ou seja, com renda insuficiente para satisfazer suas necessidades básicas (Kageyama, Hoffmann, 2006). No Brasil, os estudos são mais recentes e ganharam relevância, tanto na academia quanto entre os formuladores de políticas públicas, após a estabilização da moeda brasileira em 1994, quando a inflação deixa de ser o principal foco das atenções (Rocha, 2006).

A noção de pobreza refere-se a algum tipo de privação, que pode ser somente material ou incluir elementos de ordem cultural e social, em face dos recursos disponíveis de uma pessoa ou família. De acordo com Hagenaars e De Vos (1988), todas as definições de pobreza podem ser enquadradas numa das três categorias seguintes: a) pobreza é ter menos do que um mínimo objetivamente definido (pobreza absoluta); b) pobreza é ter menos do que outros na 
sociedade (pobreza relativa); c) pobreza é sentir que não se tem o suficiente para seguir adiante (pobreza subjetiva). Por sua vez, Sônia Rocha (2006) define a pobreza "de forma genérica como a situação na qual as necessidades não são atendidas de forma adequada”. A questão é como especificar essas necessidades de maneira adequada.

Apesar do conceito de pobreza ser universalmente aceito, ele pode variar de acordo com as normas da sociedade e as condições locais específicas. No Brasil as percepções de pobreza e as características que qualificam os pobres são diferentes, pois, entre outros fatores, possui uma ocupação desigual ao longo do território, profundas disparidades regionais, diferentes níveis de desenvolvimento e alta concentração de renda.

Diante das variações sobre as dimensões da pobreza e o que levar em consideração para defini-la, diferentes autores propõem critérios diferenciados de mensuração. De qualquer modo, as principais linhas de pobreza utilizadas nos estudos brasileiros (valor monetário que delimita o pobre do não pobre) podem ser dividas em duas tipologias: a) linhas de pobreza arbitrárias, como o valor de US\$ 1,0o diário utilizado pelo Banco Mundial, o valor de meio salário mínimo per capita ou um valor fixo, como utilizado pelo Ministério do Desenvolvimento Social; e b) a linha de pobreza a partir do consumo observado em que se levam em conta as especificidades locais em relação ao consumo de uma cesta alimentar adequado e um conjunto de necessidades básicas, como a calculada pelo IETS.

Sobre a incidência da pobreza no Brasil e com a linha de pobreza adotada pelo IETS, verificou-se que no período compreendido pelos Censos Demográficos de 1970 e 1980, houve uma forte queda na pobreza (passando de $68 \%$ da população para $35 \%$ ) em decorrência do aumento da renda. Já no decorrer da década de 1980 houve oscilações e entre o final dos anos 80 e 1994 a proporção de pobres se situou em torno de 30\%. No entanto, com a implementação do Plano Real, houve uma nova queda na pobreza que passou para 20\%, proporção essa que se manteve até o início dos anos 2000 (Rocha, 2006). Esses resultados vão de encontro ao verificado por Ramos (2004), que mesmo utilizando outra metodologia afirma o declínio da pobreza entre 1980 e 2000 em sete pontos percentuais, passando de $40 \%$ para $33 \%$, principalmente após o plano de estabilização monetária.

Por sua vez, em meados dos anos 2000 há certo consenso na literatura econômica, independentemente da linha de pobreza utilizada, que houve fortes sinais de queda na pobreza, decorrentes tanto do aumento da renda, como pela diminuição da desigualdade social, fato até então inédito no Brasil (Neri, 2006, Hoffmann, 2006, IPEA 2012, Rocha, 2013). Essa queda na pobreza mais recente é vista no Gráfico 1 que utiliza como linha de pobreza a partir do consumo observado (calculada pelo IETS) para evidenciar a evolução da pobreza tanto no Brasil como no Paraná. Nota-se, que tanto e estado analisado quanto o país apresentam, de maneira geral, a mesma tendência. A despeito 
disso, se em 1992 a porcentagem de pobres no Brasil e no Paraná próxima a 46\%, em 2011 este último apresentava cerca de 10\% de sua população na pobreza, enquanto que no Brasil a incidência de pobres representa o dobro desse valor, ou seja, a queda da pobreza no estado foi mais intensa que o verificado no em todo o país.

\title{
GRAFICO 1: PORCENTAGEM DE POBRES NO BRASIL E NO PARANÁ
}

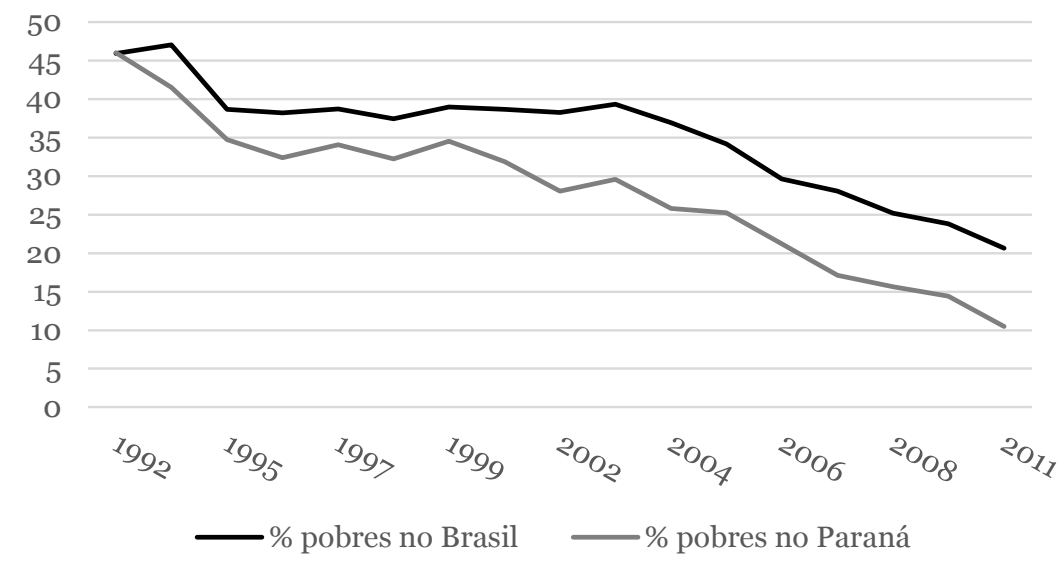

Fonte: Elaboração própria com dados do IETS (2012)

IPEA (2012) sintetiza a queda na pobreza do Brasil e afirma que o país, em um período menor que o previsto cumpriu as metas do milênio firmadas junto à ONU concernentes à redução da pobreza:

\begin{abstract}
Como consequência da manutenção do crescimento com redução da desigualdade, a pobreza mantém uma contínua trajetória decrescente, que vem desde o fim da recessão de 2003 independentemente da linha de pobreza e da medida usada. Olhando para a década como um todo, se usarmos a medida utilizada no programa Bolsa Família mantida em termos reais ou as linhas internacionais de pobreza e de extrema pobreza das metas do milênio da ONU, todas caíram mais de $55 \%$ entre 2001 e 2011. Desta forma, o Brasil cumpriu o compromisso de um quarto de século em um decênio. Ou seja, se fez mais que o previsto para 25 anos em menos de 10 anos. (IPEA, 2012, p.8)
\end{abstract}

Especificamente para este estudo e considerando a indisponibilidade de se obter linhas de pobrezas a partir do consumo para cada município paranaense, utilizou-se o critério utilizado pelo MDS e, por extensão, pelo Programa Bolsa Família (PBF), em que o domicílio é considerado pobre caso o rendimento mensal per capita seja igual ou inferior a $\mathrm{R} \$ 140,00$. 


\section{Metodologia}

A metodologia está dividida em dois blocos: no primeiro será exposta a explicação das variáveis utilizadas, enquanto no segundo estarão evidenciados o instrumental de análises de dados espaciais e os modelos econométricos espaciais.

\subsection{Descrição dos Dados}

Usaram-se dois tipos de dados nesse trabalho. O primeiro refere-se à malha de municípios georreferenciada do Paraná (arquivo shapefile), disponível no site do Instituto Paranaense de Desenvolvimento Econômico e Social IPARDES. A segunda consiste na base das informações estatísticas extraídas do Censo Demográfico do ano de 2010 para os municípios paranaenses (no caso da Pobreza) e no site do IPARDES (para os demais indicadores). Para a manipulação dos dados juntamente com o mapa foi utilizado o software Geoda 1.2.0.

Foram utilizados os seguintes indicadores:

Pobreza: razão entre o número de moradores em domicílios particulares com rendimento mensal per capita de até $\mathrm{R} \$ 140,00$ e o total de moradores em domicílios particulares. Esse valor da linha de pobreza foi estipulado pelo MDS, sendo utilizado como parâmetro para a concessão dos benefícios do Programa da Bolsa Família;

Renda Média Domiciliar per Capita: Média das rendas domiciliares per capita das pessoas residentes no município, sendo a renda domiciliar per capita a soma dos rendimentos mensais dos moradores do domicílio, em reais, dividida pelo número de seus moradores;

Grau de Urbanização: Percentagem da população da área urbana em relação à população total;

Índice de Gini da Renda Domiciliar per Capita: Mede o grau de concentração da distribuição de renda domiciliar per capita de uma determinada população e em um determinado espaço geográfico. Interpretação: Quando o índice tem valor igual a um (1), existe perfeita desigualdade, isto é, a renda domiciliar per capita é totalmente apropriada por um único indivíduo. Quando ele tem valor igual à zero (o), tem-se perfeita igualdade, isto é, a renda é distribuída na mesma proporção para todos os domicílios. Quanto mais próximo da unidade, maior a desigualdade na distribuição de renda.

A Tabela 1 mostra a descrição das variáveis quanto aos seus valores médios e extremos. Chama à atenção a alta amplitude em todas as variáveis e o fato de todas as variáveis apresentam valores máximos bem acima da média. 
TABELA 1: DESCRIÇÃO DAS VARIÁVEIS

\begin{tabular}{lccc}
\hline Variáveis & Média & Máximo & Mínimo \\
\hline Pobreza (\%) & 9,4 & 25,3 & 1,3 \\
Renda média (R\$) & 600 & 1.536 & 274 \\
Urbanização (\%) & 68 & 100 & 9 \\
Índice de Gini & 0,47 & 0,64 & 0,33 \\
\hline
\end{tabular}

Fonte: Dados do IPARDES e do IBGE. Elaboração própria.

\subsection{Econometria Espacial}

Esse artigo utiliza como metodologia o instrumental de econometria espacial, uma vez que os modelos tradicionais de regressão linear não levam em consideração as consequências de autocorrelação e heterogeneidade espaciais. Desse modo, a econometria espacial é utilizada quando uma variável de uma determinada localidade influencia a mesma (ou outra) característica em uma localidade geograficamente próxima. Para tanto, essa seção será dividida em 3 partes: a) Matriz de Dados Espaciais; b) Análise Exploratória de Dados Espaciais (AEDE); c) Modelos Econométricos Espaciais (MEE).

\subsubsection{Matriz de Dados Espaciais}

O estudo de econometria espacial começa com a representação dos dados espaciais na forma matricial, em que os vizinhos de cada localidade são especificados por meio da matriz de pesos especiais “ $W$ ”. Dessa forma, para cada ponto do espaço, é definido um conjunto de vizinhança que interage com ele.

Um dos principais métodos de ordenar as informações ao longo do espaço é a utilização do critério de contiguidade (vizinhança), que reflete a posição de uma unidade em relação às demais unidades no espaço. Quanto à dependência espacial, pressupões-se que regiões vizinhas apresentam um grau maior de dependência do que as demais. Os dois critérios de contiguidade mais utilizados são a "Rainha" e a "Torre" em alusão aos movimentos do jogo de xadrez. Por exemplo, com o critério Rainha na Figura 1, a região 5 tem como vizinhos todas as demais regiões que estão representadas, enquanto que o critério Torre considera como vizinhos de 5 as regiões 2, 4 e 6. Desse modo, o critério Rainha considera uma região vizinha se essa possui fronteiras ou vértices comuns, enquanto que o critério Torre considera como vizinho, somente os locais com fronteira em comum. 
FIGURA 1: MAPA ESTILIZADO DE VIZINHANÇA

\begin{tabular}{|c|c|c|}
\hline 1 & 2 & 3 \\
\hline 4 & 5 & 6 \\
\hline
\end{tabular}

Fonte: Elaboração própria.

Os dados de vizinhança são representados na forma de uma matriz simétrica, em que $a_{i j}$ (elemento que representa a relação de vizinhança entre o espaço $i$ e o espaço $j$ assume o valor de $1\left(W_{i j}=1\right)$ se os locais são vizinhos e valor o $\left(W_{i j}=0\right)$, caso não sejam. Por exemplo, o elemento a11 relata a informação de vizinhança da região 1 com ela mesma e, como uma região não é vizinha de si mesma, recebe o valor 0 .

Existem outras maneiras de criar uma matriz $W$, sendo uma delas a matriz de $\mathrm{k}$ vizinhos mais próximos, em que a distância entre os locais é mais importante que a existência da fronteira geográfica em si.

Logo, a tarefa é encontrar a matriz de pesos espaciais que melhor representa a verdadeira correlação espacial dos dados estudados. Uma forma bastante utilizada de escolhê-la é utilizar a matriz que apresenta o maior valor de autocorrelação entre os dados, assunto que será abordado na próxima seção.

\subsubsection{Análise Exploratória de Dados Espaciais (AEDE)}

Criada a matriz de pesos espaciais, como verificar se os dados apresentam dependência espacial, como também heterogeneidade espacial. Para responder a essa pergunta se utiliza a AEDE, cuja importância está na descrição da distribuição espacial, dos padrões de associação espacial (clusters) e identificação dos outliers. Com esse método, consegue-se extrair medidas de autocorrelação espacial global e local, além de investigar a influência dos efeitos espaciais.

O primeiro passo no estudo da AEDE é verificar a presença de aleatoriedade dos dados espaciais. Se os dados são aleatórios, o valor de uma variável em um local não depende dos valores dessa variável nos locais vizinhos. Nesse caso, não faz sentido a utilização da econometria espacial. Desse modo, a AEDE deve preceder a modelagem econométrica e indicará se é necessário utilizar a econometria espacial.

Para verificar se dos dados espaciais são aleatórios, faz-se duas análises: a) autocorrelação espacial global e b) autocorrelação espacial local.

Para calcular a autocorrelação espacial global, faz-se a estatística do Índice de Moran $(I)$, sendo que a hipótese nula se refere à aleatoriedade espacial. (Almeida, 2004). 


$$
I=\left(\frac{n}{s_{0}}\right)\left(\frac{z^{\prime} W z}{z^{\prime} z}\right)
$$

O Sendo n o número de unidades espaciais, $z$ o vetor de n observações e $W$ a matriz de pesos espaciais utilizada. So representa o somatório dos elementos da matriz de pesos espaciais $W$.

O $I$ de Moran varia entre -1 e 1 e fornece uma medida de associação linear entre os vetores $Z_{t}$ e a média ponderada dos valores da vizinhança $\left(W Z_{t}\right)$. Como o $I$ de Moran tem um valor esperado de $-\left[\frac{1}{(n-1)}\right]$, valores próximos de zero indicam a ausência de um padrão espacial dos dados, ou seja, a inexistência de autocorrelação espacial. Uma vez que o valor calculado seja superior ao valor esperado, há uma indicação de autocorrelação espacial positiva, ou seja, se uma variável estudada apresentar valor alto (baixo) em um local apresentará valor alto (baixo) da mesma variável nas localizações vizinhas. Por sua vez, se o $I$ de Moran calculado for inferior ao valor esperado, há a indicação de autocorrelação negativa. De acordo com Almeida (2004), autocorreção espacial positiva indica que a característica estudada apresenta similaridade entre seus valores, enquanto que a autocorrelação espacial negativa indica dissimilaridade entre os valores.

No entanto, a autocorrelação global pode omitir padrões do comportamento da variável nos níveis locais estatisticamente significantes, de modo que foram criados os índices de autocorrelação espacial local. Esses indicadores tem a capacidade de detectar regimes de associação espacial diferenciado porque produzem um valor específico para cada área. O mais conhecido deles é o Índice de Moran Local:

$$
I_{i}=\frac{\left(y_{i}-\bar{y}\right) \sum_{j} w_{i j}\left(y_{i}-\bar{y}\right)}{\sum_{i} \frac{\left(y_{i}-\bar{y}\right)^{2}}{n}}
$$

Se $I$ de Moran Local maior que o, há indicação de clusters com valores similares ao redor de $i$; $I$ de Moran menor que o, clusters de valores diferentes ao redor de i; enquanto que I de Moran Local igual a o, indica ausência de clusters.

O diagrama de dispersão de Moran é uma forma adicional de verificar a dependência espacial. Através da representação do coeficiente da regressão, com valores normalizados sobre a média dos vizinhos, é possível visualizar a correlação linear entre $z$ (variável normalizada) e $W z$ (média dos vizinhos).

O diagrama de dispersão de Moran é dividido em quatro quadrantes. $\mathrm{O}$ primeiro quadrante (Q1) mostra os locais com altos valores para a variável analisada, cercadas por locais com valores acima da média para essa variável. 
Esse quadrante é comumente chamado de Alto-alto (AA). O Q2 representa os locais com baixo valor para determinada variável, cercados por locais com altos valores - Baixo-alto (BA); o Q3 representa os locais com baixos valores cercado por locais com baixos valores também - Baixo-baixo (BB); enquanto que o Q4 representa os locais com valores altos e cercados por locais com valores baixo - Alto-baixo (AB).

\section{FIGURA 2: GRÁFICO DE DISPERSÃO}

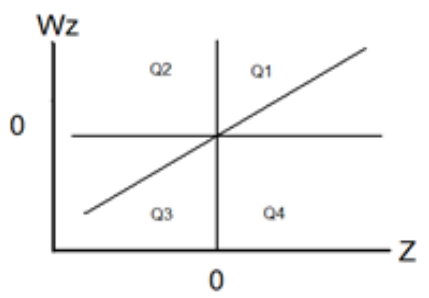

Desse modo, os locais que estão nos quadrantes 1 e 3 denotam pontos com associação espacial positiva, ou seja, o local e a média da vizinhança apresenta, valores semelhantes. As regiões com essas características formam clusters com valores similares. Por sua vez, os quadrante 2 e 4, apresentam autocorrelação espacial negativa. Em oposição aos clusters tem-se a ocorrência dos Outliers, caracterizado pelas observações que não seguem o processo de dependência espacial predominante da dependência espacial dos demais dados.

\subsubsection{Modelos econométricos espaciais}

Após a Análise Exploratória dos Dados Espaciais (AEDE), caso se confirme a presença de dependência espacial, é necessária, então, a estimação dos modelos econométricos espaciais. Esses diferem dos modelos "tradicionais", uma vez que incorporam o componente espacial. Esses componentes se referem a defasagens na variável dependente, defasagens na variável independente e/ou defasagens no termo de erro.

A autocorrelação espacial, assim como ocorre no caso da autocorrelação temporal, implica em prejuízos na estimação do modelo pelo método de Mínimos Quadrados Ordinários (MQO). Se a autocorrelação ocorre na variável dependente, as estimativas de MQO são viesadas e inconsistentes. Por sua vez, quando a correlação está presente no termo do erro, não há viés, sem inconsistência, mas o estimador de MQO deixa de ser o mais eficiente. Apesar dessas deficiências, é preciso primeiramente estimar o modelo MQO, através do qual serão feitos testes que indicarão o modelo econométrico espacial mais apropriado.

Os testes feitos no modelo estimado por MQO para detectar a autocorrelação espacial são divididos em duas categorias: os testes gerais e os testes 
específicos. Os primeiros indicam a presença de autocorrelação, contudo não detectam qual é o tipo de autocorrelação espacial predominante. Ao passo que os testes específicos indicam o tipo predominante de autocorrelação presente na regressão.

O primeiro teste geral para identificação de autocorrelação é a Estatística $I$ de Moran, uma adaptação do teste $I$ de Moran para o contexto da análise de regressão linear. A hipótese nula do teste assume que os resíduos da regressão estimada por MQO são distribuídos aleatoriamente ao longo do espaço. A rejeição da hipótese nula sugere que os resíduos são autocorrelacionados espacialmente.

No que diz respeito aos testes específicos, a maior parte deles é do tipo Multiplicador de Lagrange (ML). O Teste $M L O$ e analisa a defasagem espacial, com a hipótese nula de $H_{0}: \rho=0$. Enquanto que o Teste $M L \lambda$ investiga a autocorrelação espacial no erro e tem como hipótese nula $H_{0}: \lambda=0$.

O procedimento para indicar o modelo econométrico mais apropriado é: a) estimar o modelo pelo método de $\mathrm{MQO}$; b) testar a presença de autocorrelação através das estatística $M L \rho$ e $M L \lambda$; c) caso os dois testes não sejam significantes, o modelo não apresenta autocorrelação espacial e, dessa forma, o modelo "tradicional" é o mais apropriado; d) caso os dois testes sejam significantes, calcula-se as versões robustas desses testes, $M L^{*} \rho$ e $M L^{*} \lambda$ e estima-se o modelo mais significante; e) caso apenas um dos testes seja significante, o modelo mais adequado é que foi significante.

A seguir serão expostos os principais modelos econométricos espaciais: a) defasagem espacial, b) o modelo de erro espacial e c) cruzado espacial.

\section{a) Modelo por Defasagem Espacial (MDE)}

Esse modelo é representado por:

$$
Y=\rho W_{1} Y+\beta_{1}+\beta_{2} X+\varepsilon
$$

Em que $W_{1} Y$ é a variável dependente defasada pela matriz de pesos espaciais, $\rho$ é o coeficiente autorregressivo espacial, que mede a influência média dos vizinhos sobre a observação no vetor $y$, indicando a proporção da variação total em y explicada pela dependência espacial. Esse modelo não pode ser estimado por MQO devido à endogeneidade do regressor $W y$. Por isso, é estimado via máxima verossimilhança.

Em relação ao MQO, esse modelo possui um termo a mais para capturar o efeito da vizinhança.

\section{b) Modelo por Erro Espacial (MEE)}


Esse modelo é apropriado quando as variáveis não incluídas no modelo e presentes no componente do erro são autocorrelacionados espacialmente. É representado por:

$$
Y=\beta_{1}+\beta_{2} X+\mu
$$

em que $\mu=\lambda W \mu+\varepsilon$

Sendo o coeficiente $\lambda$ o parâmetro do erro autorregressivo espacial que acompanha a defasagem $W \mu$. Assim como o modelo anterior, o MEE é estimado via máxima verossimilhança.

\section{c) Modelo Regressivo Cruzado Espacial}

Esse modelo considera que pelo uma das variáveis explicativas tenham seus efeitos espraiados nas regiões vizinhas, além e apresentar defasagem espacial para a variável dependente. A representação formal do modelo é dada por:

$$
Y=\beta X+W X \tau+\varepsilon
$$

Sendo que o $\tau$ representa o efeito transbordamento.

Esse modelo pode ser estimado por MQO sem incorrer em problemas.

\subsubsection{Modelo proposto}

O modelo a ser estimado pode ser formalmente, representado por:

$$
\text { Pobreza }=\beta_{1}+\beta_{2} \text { Gini }+\beta_{3} \text { Urbanização }+\beta_{4} \text { Renda Média }+\varepsilon
$$

As variáveis do modelo já foram apresentadas anteriormente. É importante destacar que se espera que a pobreza sofra um efeito positivo do Gini e um efeito negativo da urbanização e da renda. A equação (1) será estimada tanto por MQO quanto pelas técnicas de econometria espacial de modo a verificar se os efeitos espaciais são significativos para estudos sobre a pobreza.

\section{Resultados e discussões}

Uma vez visto, no capítulo anterior, a metodologia utilizada na econometria espacial, esse capítulo utilizará esse instrumental para estudar a pobreza nos municípios paranaenses. Será analisada a distribuição espacial da pobreza entre os municípios, além de verificar se a utilização da econometria espacial é conveniente ou não para o estudo proposto e, caso sim, qual o modelo econométrico espacial é o mais apropriado. Por fim, será estimado o modelo. 


\subsection{Resultados da Análise Exploratória dos Dados Espaciais (AEDE)}

A distribuição geográfica da pobreza no estado do Paraná é ilustrada na Figura 3. A figura mostra claramente que as cidades com a maior porcentagem do número de moradores em domicílios com rendimento mensal per capita abaixo da linha da pobreza, que chamaremos de "Pobreza" se encontram na região centro-sul e no litoral sul do estado, enquanto que as menores taxas estão em algumas cidades da região metropolitana de Curitiba e no noroeste do Estado. Dos 399 municípios, 12 apresentam taxas de pobreza acima de $20 \%$, enquanto que 87 apresentam taxas abaixo de $5 \%$.

De acordo com a Figura 3 nota-se que a região centro-sul do estado, área mais escura do mapa, concentra a maior parte dos municípios mais pobres do estado. De acordo com os dados do IPARDES (2010), entre os dez municípios paranaenses com os maiores porcentuais de domicílios pobres, sete estão localizados na região centro-sul do estado. Por outro lado as regiões norte central e oeste, regiões mais claras do mapa, encontram-se os municípios de menor pobreza. Outra região que merece destaque é a região Metropolitana de Curitiba, onde se encontram os municípios de maior e menor rendimento domiciliar mensal per capita do estado, respectivamente, Curitiba com $\mathrm{R} \$ 1.536$ e Doutor Ulysses com $\mathrm{R} \$ 274$.

FIGURA 3: POBREZA POR MUNICÍPIO PARANAENSE, PROPORÇÃO DE POBRES.
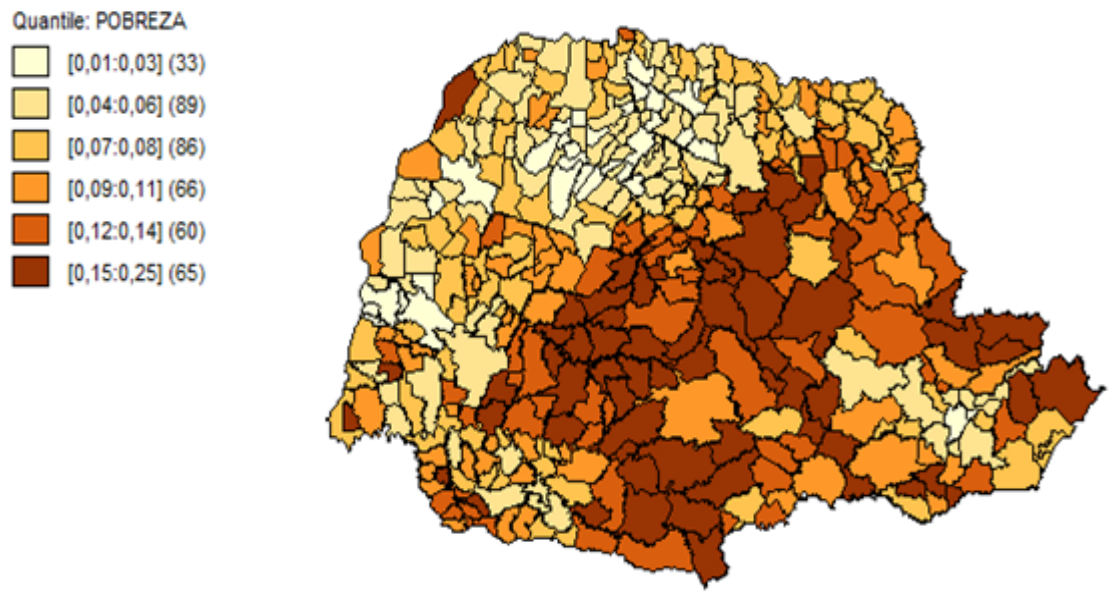

Fonte: Elaboração própria com o uso do Geoda.

De acordo com a literatura regional, vários são os motivos que explicam o alto índice de pobreza da região centro-sul do estado. O primeiro motivo tem motivações políticas, tendo em vista a emancipação precoce sem a devida infraestrutura e fontes de receita para ampliar e melhorar as condições de 
vida de seus habitantes. Segundo, talvez o mais difundido na literatura para explicar o alto índice de pobreza na região, é o baixo índice de urbanização, ou seja, grande parte dos habitantes vive na zona rural, porém a estrutura agrícola desta região baseada em latifúndios, extrativismo e subsistência intensifica os problemas relacionados com a pobreza.

Analisando os dados do IPARDES (2010), destaca-se negativamente o município de Laranjal situado no centro-sul do estado, com 6.360 habitantes, possui o maior percentual de pobreza do estado, 25,3\%, e o terceiro menor rendimento domiciliar mensal per capita do estado, além de mais de $68 \%$ de sua população residindo no campo. Nesta região com altas taxas de pobreza evidencia-se também os municípios de Inácio Martins, Nova Laranjeiras, Goioxim, Coronel Domingos Soares, Mato Rico e Rio Bonito do Iguaçu. A taxa média de pobreza nesses municípios é de 22,0\%, correspondendo a 134,8\% superior a taxa média de pobreza do estado do Paraná que é de 9,4\%.

A região norte central é caracterizada pela maior quantidade de municípios com menor taxa de pobreza. Desde a cultura do café, meados da década de 70 , até o segundo maior parque industrial do estado e uma forte presença do setor de serviços, a região norte central sempre apresentou dados econômicos de destaque no estado. Entre os dez municípios paranaenses com os menores porcentuais de taxa de pobreza, três estão localizados nesta região. Destacam-se os municípios de Maringá, Floraí e Miraselva. A taxa média de pobreza destes municípios é $1,9 \%$ bem abaixo da média estadual. Nesta região encontram-se também o segundo e a quarto melhor rendimento domiciliar mensal per capita do estado, respectivamente, Maringá com $\mathrm{R} \$ 1.187,53$ e Londrina com $\mathrm{R} \$ 1.062,64$.

Outra região que merece destaque é a região oeste, uma vez que seis dos dez municípios com melhor rendimento domiciliar mensal per capita do estado estão localizados nesta região: Quatro Pontes, Marechal Cândido Rondon, Itaipulândia, Entre Rios do Oeste, Serranópolis do Iguaçu e Cascavel. O rendimento domiciliar mensal per capita médio dos municípios citados é de $\mathrm{R} \$ 1.020,62$ correspondendo a 41,20\% superior ao rendimento domiciliar mensal per capita médio do estado que é de $\mathrm{R} \$ 600,04$. Nesta região encontra-se também o município com menor taxa de pobreza do estado, o município de Quatro Pontes com 1,3\%.

Logo, a Figura 3, parece indicar uma semelhança na taxa de pobreza entre os municípios vizinhos. Contudo, é necessário fazer os cálculos de dependência espacial para comprovar essa afirmação.

\section{2 Índice de Moran Global}

Como foi visto na metodologia, antes de calcular o índice de Moran Global propriamente dito, é preciso verificar qual a matriz de pesos espaciais melhor 
se adapta aos dados de pobreza disponíveis. Desse modo, foram calculadas diferentes matrizes, conforme evidenciado na Tabela 2. Em todas as matrizes de pesos, foram encontrados valores positivos e estatisticamente significantes, indicando a existência de autocorrelação espacial positiva para todas as matrizes utilizadas, ou seja, nota-se uma similaridade localizacional nos valores da variável.

Como a matriz de ordem espacial "Rainha" foi a que apresentou maior valor de I de Moran Global, é essa matriz que será utilizada nos demais testes, como também as estimações dos modelos econométricos utilizando essa matriz de pesos.

TABELA 2: COEFICIENTE DO I DE MORAN GLOBAL PARA A POBREZA NO PARANÁ

\begin{tabular}{lrr}
\hline & Valor & P-valor \\
\hline Rainha de ordem 1 & 0,66107 & 0,001 \\
Torre de ordem 1 & 0,66102 & 0,001 \\
4 vizinhos mais próximos & 0,6459 & 0,001 \\
5 vizinhos mais próximos & 0,63376 & 0,001 \\
10 vizinhos mais próximos & 0,59814 & 0,001 \\
\hline
\end{tabular}

Fonte: Elaboração própria

O primeiro dos testes é o diagrama de dispersão do I de Moran Global, na Figura 4, onde se verifica que o I de Moran Global de o,66107 excede o seu valor esperado e, desse modo, rejeita-se a hipótese nula de que a distribuição da pobreza nos municípios paranaenses segue uma distribuição aleatória. Consequentemente, o estudo da influência espacial da pobreza é pertinente.

FIGURA 4: I DE MORAN GLOBAL

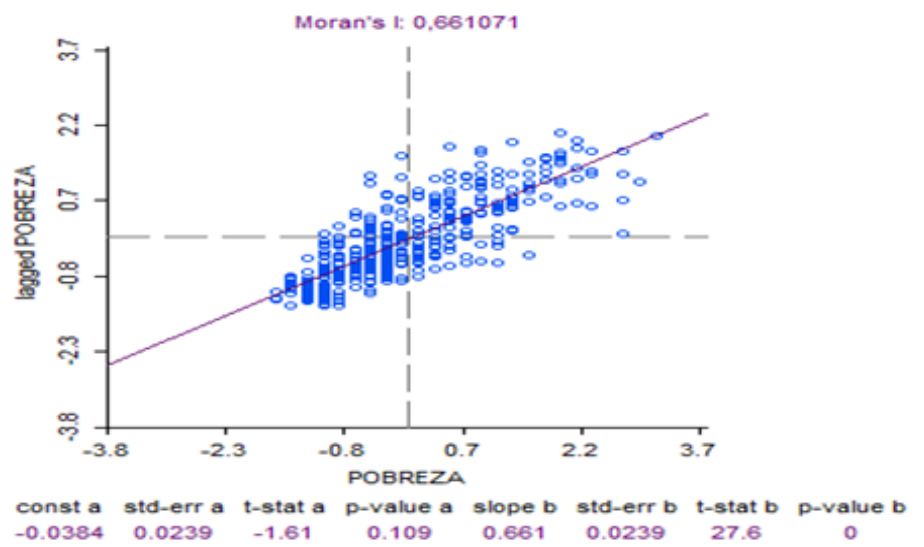

Fonte: elaboração própria utilizando o Geoda. 
Ao verificar a dispersão das variáveis entre os quadrantes, nota-se que os municípios estão, predominantemente, nos quadrantes I e III, ou seja, nos quadrantes em que um município com alto índice de pobreza está cercado por municípios com altas taxas de pobreza, o mesmo ocorrendo com municípios com baixa pobreza, cercado por municípios com o mesmo atributo.

Da mesma forma, o I de Moran Global univariado das variáveis explicativas, conforme a Tabela 3 mostra que a distribuição mostra que a distribuição das variáveis explicativas Urbanização, Renda Média e Índice de Gini apresentam influência espacial estatisticamente significativas.

Por sua vez, o I de Moran bivariado faz a comparação de duas variáveis do modelo e seus resultados são apresentados na Tabela 3. Para tanto, a pobreza (variável endógena) é comparada com cada uma das variáveis exógenas.

Em relação às variáveis Urbanização e Renda Média, os Is de Moran bivariados indicam que essas variáveis estão, predominantemente, nos quadrantes II e IV, de modo que os municípios mais pobres estão cercados por municípios com baixos valores de Urbanização e Renda Média (Q4), enquanto que os municípios menos pobres estão rodeados por municípios com maior renda média e mais urbanizados. No que diz respeito ao Índice de Gini, o resultado difere das variáveis anteriores, uma vez que há predominância de municípios pobres com altos níveis de concentração de renda (Q1) e municípios menos pobres, com Índices de Gini menores.

TABELA 3: ESTATÍSTICA I DE MORAN GLOBAL DAS VARIÁVEIS ESTUDADAS

\begin{tabular}{lrrrr}
\hline Variável & $\begin{array}{c}I \text { de Moran } \\
\text { univariado }\end{array}$ & p-valor & $\begin{array}{l}I \text { de Moran } \\
\text { bivariado* }\end{array}$ & p-valor \\
\hline Pobreza & 0,661 & 0,001 & & \\
Grau de Urbanização & 0,4111 & 0,001 & $-0,4308$ & 0,001 \\
Renda Média & 0,2946 & 0,001 & $-0,3955$ & 0,001 \\
Índice de Gini & 0,3715 & 0,001 & 0,4376 & 0,001 \\
\hline
\end{tabular}

* entre a variável dependente Pobreza e a variável explicativa.

p-valor baseado em 999 permutações aleatórias. Fonte: elaboração própria.

Por fim, conclui-se que as variáveis explicativas apresentam impacto espacial na taxa de pobreza municipal, já que todos os Is de Moran bivariados foram estatisticamente significantes.

\section{3 Índice de Moran Local}

A Figura 5 com o Índice de Moran Local auxilia na localização dos clusters. É possível verificar três regiões com dependência espacial estatisticamente 
significante. Duas regiões apresentam o comportamento do tipo baixo-baixo (Q3), situam-se, basicamente nas regiões norte-central e oeste do Paraná. Por sua vez, a terceira região de concentração de clusters que compreende 70 municípios em uma área geográfica extensa, está localizada na região centro-sul a se estende para uma parte do litoral, apresenta o padrão do tipo alto-alto (Q1).

\section{FIGURA 5: MAPA DE CLUSTERS DA POBREZA NO PARANÁ EM 2010}
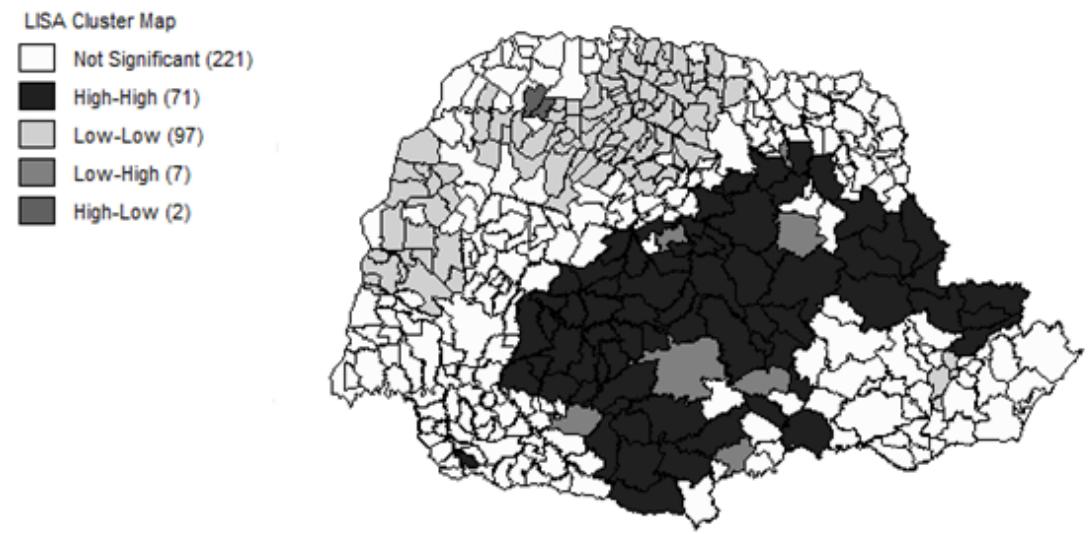

Fonte: Elaboração própria.

Para uma melhor verificação do impacto das variáveis explicativas regiões vizinhas na pobreza dos municípios, a Figura 6, mostra o resultado do I de Moran Local bivariado, estando a pobreza no eixo X e cada uma das variáveis no eixo $\mathrm{Y}$.

O mapa que mais chama a atenção é o que confronta a pobreza com o Índice de Gini, no qual nota-se duas grandes regiões, uma com municípios com alta pobreza e cercados por vizinhos com alto índice de Gini representado em cor mais escura (região centro-sul) e outra em que municípios com baixa pobreza são cercados por municípios com baixo índice de Gini, região de cinza claro.

Em relação à urbanização, também há duas regiões de clusters bem nítidas no mapa: uma região do norte central (onde está Maringá e Londrina) que apresentam baixos índices de pobreza com elevado grau de urbanização na vizinhança (low-hight) e uma região central no qual há alta pobreza e baixa urbanização dos vizinhos. Por sua vez, a região central também apresenta uma concentração de municípios com alta pobreza e baixa renda média na vizinhança, ainda que a área do cluster desse mapa seja menor que a dos mapas anteriores.

Por fim, os três mapas da Figura 6, assim como os demais mapas anteriores, apresenta uma cidade que "destoa” de sua vizinhança, é a cidade de Guarapu- 
ava, na região centro sul. Essa cidade, a mais populosa da região centro-sul, apesar de estar numa região de pobreza, apresenta a proporção de pobres por volta de $10 \%$. Além disso, Guarapuava apresenta um baixo índice de Gini e é cercada por vizinhos pobres.

FIGURA 6: CLUSTERS BIVARIADOS ENTRE A POBREZA E AS VARIÁVEIS EXPLICATIVAS
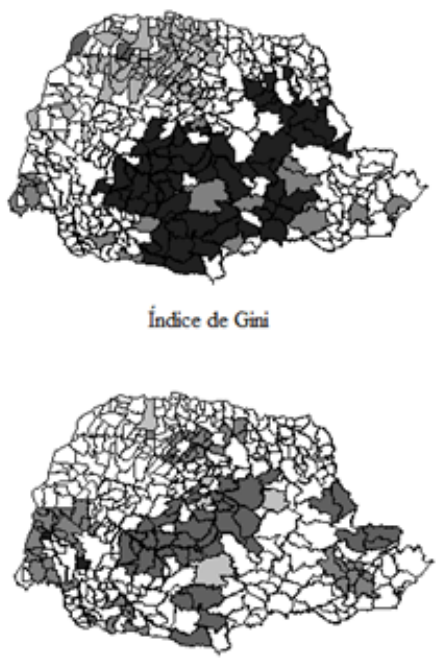

Renda Média
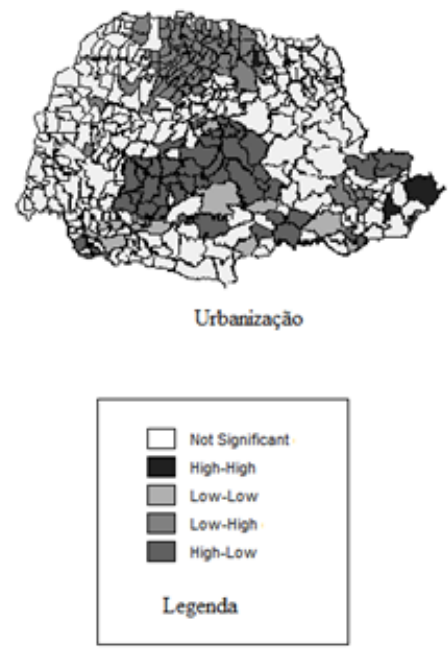

Fonte: Elaboração própria.

Dada a concentração geográfica verificada para a pobreza nos municípios paranaenses obtidos através de vários testes, a seguir estimar-se-á as equações de pobreza com os métodos econométricos espaciais.

\subsection{Resultados do Modelo econométrico}

Os modelos a seguir foram estimados com as variáveis explicativas anteriormente anunciadas. Os valores de Pobreza, Gini e Urbanização foram transformados em valores de o a 100, para facilitar a análise.

A Tabela 4 apresenta os resultados do modelo estimado por MQO. Os resultados confirmam os sinais esperados. Como todos os coeficientes foram estatisticamente significantes a 1\%, pode-se dizer que um aumento na variável Índice de Gini causa um aumento na taxa de pobreza. Enquanto isso, um aumento na taxa de urbanização e na renda média contribuem para diminuir a pobreza. 


\begin{tabular}{lcccc}
\hline Variável & Coef. & desvio-padrão & $\mathrm{t}$ & $\mathrm{p}$-valor \\
\hline Constante & 5,9118 & 1,0153 & 5,82 & 0,001 \\
Gini & 0,4291 & 0,0192 & 22,34 & 0,001 \\
Urbanização & $-0,0476$ & 0,0064 & $-7,38$ & 0,001 \\
Renda Média & $-0,0226$ & 0,0008 & $-26,36$ & 0,001 \\
R2 & 0,83 & & & \\
Teste F & 686,47 & & & \\
Prob (F) & 0,001 & & & \\
Log-likelihood & $-842,18$ & & & \\
Condition Number & 26,68 & & & \\
Critério Akaike & 1692,37 & & & \\
Critério Schawarz & 1708,32 & & & \\
Jarque-Bera & 0,001 & & & \\
Breush-Pagan & 0,001 & & & \\
White & 0,001 & & & \\
\hline
\end{tabular}

Fonte: Elaboração própria

O teste R2 indica que as variáveis explicativas explicam 83\% da pobreza no Estado. Contudo, o teste Jarque-Bera que analisa a normalidade dos erros indica que nesse caso, os erros não seguem uma distribuição normal, fato que pode causar problemas na estimação, apesar do teste "Condition Number" com valores inferiores a 30 indicarem a não existência de multicolinearidade. Por fim, ao analisar o teste Breush-Pagan que tem como hipótese nula a homocedasticidade, nota-se que é possível rejeitar com $1 \%$ de significância a hipótese nula, ou seja, o modelo apresenta o problema da heterocedasticidade, fato bastante corriqueiro nos estudos de econometria espacial.

\subsubsection{Modelos econométricos espaciais}

Estimado o modelo por MQO na seção anterior, estimar-se-á agora por métodos espaciais. Como visto na metodologia, antes é necessário detectar a ocorrência de auto-correlação espacial e verificar qual é o modelo espacial mais adequado, com a utilização de testes gerais e específicos a partir do resultado estimado por MQO, conforme se verifica na Tabela 5 .

Como os testes dos Multiplicadores de Lagrange, tanto o de defasagem como o de erro ( $M L \rho$ e $M L \lambda)$ mostraram-se estatisticamente significantes a $1 \%$, é possível afirmar que o método de Mínimos Quadrados Ordinários não é o mais apropriado. Desse modo, é preciso verificar as versões robustas dos dois testes $\left(M L^{*} \rho\right.$ e $\left.M L^{*} \lambda\right)$ e escolher como modelo apropriado o que for mais 
significante. Uma vez que $M L \rho$ é significante a $1 \%$, enquanto que o teste $M L \lambda$ não é significante nem a 10\%. Assim, o modelo de defasagem espacial é o mais apropriado. Sendo esse representado por:

Pobreza $=\rho W_{1}$ Pobreza $+\beta_{1}+\beta_{2}$ Gini $+\beta_{3}$ Urbanização $+\beta_{4}$ Renda Média $+\varepsilon$ (5)

Em que $W_{1}$ Pobreza a variável dependente defasada pela matriz de pesos espaciais.

TABELA 5: DIAGNÓSTICO DE DEPENDÊNCIA ESPACIAL

\begin{tabular}{lcc}
\hline Teste & Valor & Prob. \\
\hline$M L \lambda$ (erro) & 19,54 & 0,001 \\
$M L^{*} \lambda$ (erro robusto) & 0,00031 & 0,98 \\
$M L \rho$ (defasagem) & 91,58 & 0,001 \\
$M L^{*} \rho$ (defasagem robusta) & 72,03 & 0,001 \\
\hline
\end{tabular}

Fonte: Elaboração própria.

Como se verifica na Tabela 6, assim como na estimação por MQO, os valores dos coeficientes estimados pelo método da defasagem espacial apresentaram o sinal esperado, ou seja, variações positivas na renda média e na urbanização tendem a resultar em variações negativas (diminuição) na taxa de pobreza, enquanto que variações positivas do Índice de Gini tendem a causar variações positivas na taxa da pobreza. Além disso, o R2 do modelo foi levemente superior com a inclusão da defasagem espacial, enquanto que persiste o problema da heterocedasticidade.

TABELA 6: RESULTADO DO MODELO DO MODELO DE DEFASAGEM ESPACIAL

\begin{tabular}{lc}
\hline & Coeficiente \\
\hline Constante & 6,02 \\
Gini & 0,293 \\
Urbanização & $-0,039$ \\
Renda Média & $-0,018$ \\
Wpobreza & 0,337 \\
R2 & 0,87 \\
Log Likelihood & $-793,21$ \\
Critério de Akaike & 1596,4 \\
Critério de Schwarz & 1616,3 \\
Breush-Pagan & 0,001 \\
\hline
\end{tabular}

OBS: Coeficientes significantes a $1 \%$.

Fonte: Elaboração Própria.

O coeficiente "novo" em relação ao modelo "tradicional” - Wpobreza - foi 
estatisticamente significante e apresentou um valor maior ao das demais variáveis, indicando um impacto maior na explicação da pobreza. Desse modo, a pobreza na vizinhança (defasagem espacial) contribui para melhor explicar a pobreza nos municípios paranaenses, ou seja, devido ao seu coeficiente positivo a maior pobreza entre os vizinhos causa uma maior pobreza nos municípios em análise.

TABELA 7: RESULTADOS DO MODELO CRUZADO

\begin{tabular}{lcccc}
\hline Variável & Coef. & desvio-padrão & $\mathrm{T}$ & p-valor \\
\hline Constante & 5,8963 & 1,416 & 4,16 & 0,001 \\
Gini & 0,2826 & 0,021 & 13,04 & 0,001 \\
Urbanização & $-0,0424$ & 0,006 & $-6,54$ & 0,001 \\
Renda Média & $-0,0174$ & 0,0009 & $-18,7$ & 0,001 \\
Wgini & $-0,0368$ & 0,054 & $-0,67$ & 0,49 \\
Wurbanização & 0,012 & 0,01 & 1,12 & 0,26 \\
Wrenda & 0,0006 & 0,002 & 0,25 & 0,79 \\
Wpobreza & 0,4364 & 0,092 & 4,72 & 0,001 \\
R2 & 0,87 & & & \\
Teste F & 400,74 & & & \\
Prob (F) & 0,001 & & & \\
Log-likelihood & $-787,47$ & & & \\
Condition Number & 49,91 & & & \\
Critério Akaike & 1590,94 & & & \\
Critério Schawarz & 1622,85 & & & \\
Jarque-Bera & 0,001 & & & \\
Breush-Pagan & 0,001 & & & \\
White & 0,001 & & & \\
\hline
\end{tabular}

Fonte: Elaboração própria.

Por sua vez, estimando o modelo econométrico espacial, em que é incorporado o efeito transbordamento das variáveis explicativas da vizinhança assim como a defasagem da variável dependente (modelo cruzado) exposto na Tabela 7 , nota-se que não houve mudanças nos valores dos coeficientes em relação às duas estimações anteriores. No entanto, os coeficientes das defasagens das variáveis explicativas possuem sinal invertido em relação a variável não defasada, porém como essas variáveis não são estatisticamente significantes a 10\%, chega-se a conclusão de que essas variáveis não contribuem para a explicação da pobreza nos municípios. Ademais, assim como no modelo anterior, no entanto com um coeficiente maior, a variável de defasagem espacial 
da pobreza (Wpobreza) é significante, indicando que a pobreza nas localidades vizinhas impacta positivamente na incidência da pobreza nas cidades paranaenses. A despeito do modelo cruzado apresentar uma falha em relação aos demais modelos, a suspeita de multicolinearidade, ("Condition Number" superior a 30), esse modelo reforça os resultados do modelo de defasagem espacial, tendo em vista que apresentaram valores próximos dos coeficientes estimados, com a manutenção dos sinais, além de cofirmar o papel relevante da variável explicativa Wpobreza.

\section{Considerações Finais}

A pobreza é uma situação que pode ser medida por diversas metodologias, desde as mais simples que determinam a linha de pobreza pela renda (unidimensional), até as mais complexas que consideram inúmeros fatores (bidimensionais). Na prática, o governo brasileiro utiliza as medidas unidimensionais como, por exemplo, a linha de pobreza adotada pelo Ministério do Desenvolvimento Social na concessão da Bolsa-Família.

Fora isso, nota-se que o tema da pobreza tem ganhado cada vez maior repercussão desde os anos 1990, com a consolidação e ampliação de programas federais e estaduais que beneficiam a população pobre, resultando não somente por isso em uma queda na porcentagem de famílias pobres no período pós Plano Real e em meados dos anos 2000.

Dessa forma o estudo mais detalhado do tema é relevante para a avaliação dos programas de combate à pobreza e $\mathrm{a}$ indicação de possíveis correções para os mesmos em direção a maior eficiência.

Ao estudar a situação da pobreza nos municípios paranaenses, ainda que esse possua com uma incidência de pobreza baixa nos últimos anos em comparação com outros Estados brasileiros, nota-se que há algumas cidades que apresentam proporção de pobres superior a 20\%. Ademais, esses municípios de acordo com a intensidade de pobreza encontram-se aglomerados, ou seja, municípios com baixo índice de pobreza estão cercados por municípios com a mesma característica e vice-versa. Pode-se dizer que a pior condição em relação à pobreza está na região centro-sul e a melhor na região norte-central e oeste do Estado. Os resultados indicaram que a influência espacial (da vizinhança) ocorre nos municípios paranaenses e é significativa, constituindo dessa forma um elemento importante no estudo da pobreza.

Com a utilização de três variáveis explicativas - Renda Média, Grau de Urbanização e Índice de Gini, verificou-se que estão espacialmente correlacionadas com a Pobreza, conforme os mapas de Clusters Bivariados, em que municípios com alta proporção de pobreza estão cercados, preponderantemente, por 
municípios com alto Índice de Gini e por baixos Graus de urbanização e baixa Renda Média, enquanto os municípios com menor pobreza possuem vizinhos com baixo Índice de Gini, alto grau de Urbanização e alta Renda Média.

Por fim, os modelos econométricos corroboraram com os resultados do I de Moran bivariado, já que em todos os modelos, quanto maior a renda média e a urbanização, menor é a incidência de pobreza, enquanto que a relação com o Índice de Gini é o inverso, quanto maior esse índice de concentração, maior a ocorrência de pobreza. No mais, os modelos econométricos com efeito espacial mostraram-se superiores para explicar a pobreza quando comparados ao modelo estimado por MQO, incorrendo que a defasagem espacial da pobreza (Wpobreza) é uma variável explicativa importante na explicação da pobreza. Além disso, os dois modelos espaciais estimados - modelo de defasagem espacial e o modelo cruzado - apresentaram resultados semelhantes resultando nos sinais esperados dos coeficientes e indicando a significância da defasagem espacial da pobreza como a variável explicativa com maior coeficiente (mais intensa), demonstrando que políticas de combate à pobreza não são eficientes, se tomadas somente ao nível municipal. Dessa forma, essas políticas devem ocorrer no âmbito regional para obterem melhores resultados.

\section{Referências Bibliográficas}

ALMEIDA, E. (2004). Curso de econometria espacial aplicada. Piracicaba: ESALQ (Material Didático).

HAGENAARS, A. \& DE VOS, K. (1998). The definition and measurement of poverty. The Journal of Human Resources, v. 23(2), pp. 211-221.

HOFFMANN, R (2006). "Queda da desigualdade da distribuição de renda no Brasil de 1995 a 2005, e delimitação dos relativamente ricos em 2005”. In: Desigualdade de Renda no Brasil: uma análise da queda recente. Brasília. IPEA.

IPARDES. Base de Dados do Paraná - BDEweb. URL [on-line]: <http://www. ipardes.pr.gov.br/imp/index.php>. Acesso em: 25 mar. 2013.

IETS- INSTITUTO DE ESTUDOS DO TRABALHO E SOCIEDADE. (2012) Distribuição de Renda Pnad 1992-2011. URL [on-line]: <http://www.iets.org.br/article. php3?id_article=1979>. Acesso em: 22 out. 2013.

KAGEYAMA, A. \& HOFFMANN, R. (2006). "Pobreza no Brasil: uma perspectiva multidimensional." Economia e Sociedade, v. 15(1), pp. 79-112.

RAMOS, L. \& MENDONÇA, R. (2004). "Pobreza e Desigualdade de Renda no Brasil.” In: Economia Brasileira Contemporânea (1945-2004). Rio de Janeiro. Ed. Campus/Elsevier.

ROCHA, S. (2006). Pobreza no Brasil. Afinal, de que se trata? 3. Ed. Editora FGV. Rio de Janeiro.

(2013). “A Pobreza no Brasil. A evolução de longo prazo (1970-2011). “ Estudos e Pesquisas $n^{\circ}$ 492. Instituto Nacional de Altos Estudos (INAE). Rio 
SILVA, L. N. S.; BORGES, M. J.; PARRÉ, J. L. Distribuição Espacial da Pobreza no Paraná

de Janeiro.

Recebido em: 17 de outubro de 2013

Aceito em: 16 de março de 2014 